\title{
Certain Types of Complex Lie Group Action
}

\author{
Ahmed Khalaf Radhi \\ Taghreed Hur Majeed \\ Department of Mathematics, College of Education \\ Al-Mustansiriya University \\ dr_ahmedk@yahoo.com \\ taghreedmajeed@yahoo.com
}

Recived : $23 \backslash 5 \backslash 2018$

Revised : 30\5\2018

Accepted : $21 \backslash 6 \backslash 2018$

\author{
Available online : $\quad 6 / 8 / 2018$
}

DOI: $10.29304 / j q c m .2018 .10 .3 .405$

\begin{abstract}
The main aim in this paper is to look for a novel action with new properties on Complex Lie Group from the Lemma of Schure, the Literature are concerned with studying the action of Lie algebra of two representations, one is usual and the other is the dual, while our interest in this work is focused on some actions on complex Lie group[10]. Let $\mathrm{G}$ be a matrix complex Lie group, and $\pi$ is representation of $G$. In this study we will present and analytic the concepts of action of complex Lie group on Hom-space. We recall the definition of tensor product of two representations of Lie group and construct the definition of action of Lie group on Hom - space, then by using the equivalent relation $\operatorname{Hom}\left(w_{2}, w_{1}\right) \cong w_{2}^{*} \otimes w_{1}$ between $\operatorname{Hom}$ and Tensor product, we get a new action : Action-complex Lie Group on tensor product. The two actions are forming smooth representation of $G$ [8], [9]. This we have new action which called triple action of Complex Lie Group $G$ denoted by TAC - complex Lie group which acting on $\operatorname{Hom}\left(\operatorname{Hom}\left(\left(w_{5} \oplus w_{4}\right), w_{3}^{*}\right), \operatorname{Hom}\left(w_{2} \oplus w_{1}, w^{*}\right)\right)$.

This $T A C$ is smooth representation of $G$. The theoretical Justifications are developed and prove supported by some concluding remarks and illustrations.
\end{abstract}

Key words : Hom - Space, Tensor Product, Action of Lie Group ,Complex Lie Group .

Mathematics subject classification: 64S40 


\section{1- Introduction}

A complex Lie group is a finite dimensional analytic manifold $G$ together with a group structure on $G$, Such that the multiplication $G x G \rightarrow G$ and attaching of an inverse $g \rightarrow g^{-1}: G \rightarrow G$ is analytic map [4],[6].

A matrix Lie group is any subgroup $G$ of $G L(n, \mathbb{C})$ with the following property [7]. If $A_{m}$ converges to some Matrix $A$, then $A \in G$ or $A$ is not invertible [5]. The Schur's lemma introduced the concepts of Lie algebra on the space of Linear maps from $W_{2}$ into $W_{1}$, which denoted by $\operatorname{Hom}\left(W_{2}, W_{1}\right)$ [1],[3]. Also introduced the concepts of action on Hom space of two representations of Lie algebr [1].

Also the main work here is to give a representation of complex Lie group by intertwine these actions (representations) and to give representation by intertwine duel of these actions (representations) and Then generalizing them.

\section{2- The TcoA of complex Lie Groups on Hom - Space}

In [2- P327], the Schur's lemma introduced the concepts of action of Lie algebra on Hom space of Two representations of Lie algebra.

\section{Lemma (2.1) [2]:}

Suppose that $\pi_{1}$ and $\pi_{2}$ are two representations of Lie algebra $g$ action on finite dimensional space $W_{1}$ and $W_{2}$ respectively . Define an Co-action of $g$ on $\operatorname{Hom}_{k}\left(W_{2}, W_{1}\right), \pi: g \rightarrow g l\left(\operatorname{Hom}_{k}\left(W_{2}, W_{1}\right)\right)$ for all $x \in g, F \in \operatorname{Hom}_{k}\left(W_{2}, W_{1}\right), \pi_{1}(x) F-F \pi_{2}(x)$ $\pi(x)(v)=\pi_{1}(x) F(v)$ and Hom $\left(W_{2}, W_{1}\right)=$ $W_{2}^{*} \otimes W_{1}$ as equivalence of representations.

\section{Lemma (2.2):}

Put $\operatorname{Hom}\left(\operatorname{Hom}\left(W_{5} \oplus W_{4}\right), W_{3}^{*}\right),\left(\operatorname{Hom}\left(W_{2} \oplus\right.\right.$ $\left.\left.W_{1}, W^{*}\right)\right)$ ) the $K-$ vector - space of all Linear maps $\left(\operatorname{Hom}\left(W_{5} \oplus W_{4}\right), W_{3}^{*}\right)$ onto $\left(\operatorname{Hom}\left(W_{2} \oplus\right.\right.$ $\left.W_{1}, W^{*}\right)$.

Define $\pi: G \rightarrow G L\left(\operatorname{Hom}\left(\operatorname{Hom}\left(W_{5} \oplus\right.\right.\right.$

$\left.\left.\left.W_{4}\right), W_{3}^{*}\right),\left(\operatorname{Hom}\left(W_{2} \oplus W_{1}, W^{*}\right)\right)\right)$,

by $\pi(a)=\pi^{*}(a) \circ F_{1} \circ\left(\pi_{2}(a) \oplus \pi_{1}(a)\right)^{-1}$ 。 $F_{2} \pi_{3}^{*}(a) \circ F_{3} \circ\left(\pi_{5}(a) \oplus \pi_{4}(a)\right)^{-1}$, $\left.W_{1}, W\right)$

$$
\text { for all } \quad a \in G, \quad F_{1} \in \operatorname{Hom}\left(W_{2}^{*} \oplus\right.
$$

$$
\begin{gathered}
F_{2} \in \operatorname{Hom}\left(W_{5} \oplus\right. \\
\left.W_{4}, W_{3}^{*}\right) \quad F_{3} \\
\in \operatorname{Hom}\left(\operatorname { H o m } \left(W_{5}\right.\right. \\
\left.\bigoplus W_{4}, W_{3}^{*}\right), \operatorname{Hom}\left(W_{2}\right. \\
\left.\left.\bigoplus W_{1}, W^{*}\right)\right) . \\
\pi(a)_{v}=\pi(a) \circ F_{1} \circ\left(\pi_{2}(a) \oplus \pi_{1}(a)\right)^{-1} \circ F_{2} \\
\circ\left(\pi _ { 3 } ( a ) \circ F _ { 3 } \circ \left(\pi_{5}(a)^{-1}\right.\right. \\
\left.\bigoplus \pi_{4}(a)^{-1}\right)_{(v)}
\end{gathered}
$$

Ahmed .K/Taghreed .H

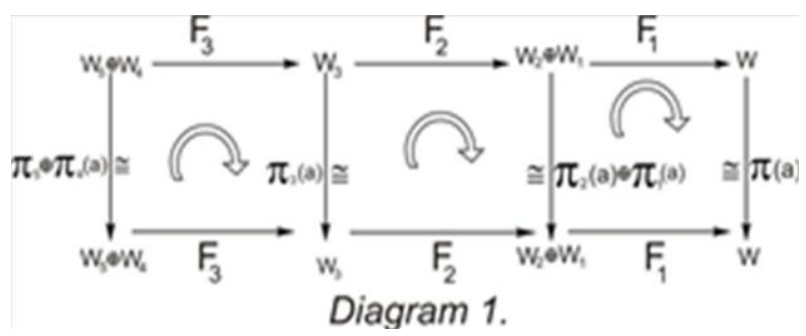

For all $a \in G, v \in\left(W_{5} \oplus W_{4}\right)$

$$
\begin{aligned}
\pi(a)_{v}=\pi(a) \circ & F_{1} \circ\left(\pi_{2}(a)^{-1} \oplus \pi_{1}(a)^{-1}\right) \circ F_{2} \\
& \circ\left(\pi _ { 3 } ( a ) \circ F _ { 3 } \circ \left(\pi_{5}(a)^{-1}\right.\right. \\
& \left.\oplus \pi_{4}(a)^{-1}\right)_{(v)}
\end{aligned}
$$

For all $a \in G, v \in\left(W_{5} \oplus W_{4}\right)$.

Where the arrow that makes the diagram 1

commutative $\pi$ is homomorphism of groups

$G$ into $\operatorname{GL}\left(\left(\operatorname{Hom}\left(W_{5} \oplus\right.\right.\right.$

$\left.\left.\left.W_{4}\right), W_{3}^{*}\right),\left(\operatorname{Hom}\left(W_{2} \oplus W_{1}, W^{*}\right)\right)\right)$.

Let $\pi_{i}: G \rightarrow G L\left(W_{i}\right)$, and

$\pi_{i}^{*}: G \rightarrow G L\left(W_{i}^{*}\right)$, for $i=1,2,3,4,5$.

The TAS of complex Lie group $G$ on

$\operatorname{Hom}_{k}\left(\operatorname{Hom}\left(\left(W_{5} \oplus W_{4}\right), W_{3}^{*}\right), \operatorname{Hom}\left(W_{2} \oplus\right.\right.$

$\left.W_{1}, W^{*}\right)$ )

is given by a representation $\pi$ such that

$\left.\pi(a)=\pi(a) \circ F_{1} \circ\left(\pi_{2}(a)^{-1} \oplus \pi_{1}(a)\right)^{-1}\right) \circ F_{2} \circ$

$\left(\pi_{3}(a) \circ F_{3} \circ \pi_{5}(a)^{-1} \oplus \pi_{4}(a)^{-1}\right)$,

For all $a \in G$.

Then the TAS of complex Lie group $G$ on

$\operatorname{Hom}_{k}\left(\operatorname{Hom}\left(\left(W_{5} \oplus W_{4}\right), W_{3}^{*}\right), \operatorname{Hom}\left(W_{2} \oplus\right.\right.$

$\left.W_{1}, W^{*}\right)$ ) is also given by representation $\pi^{*}$ such that

$$
\begin{aligned}
\pi^{*}(a)=\pi^{*}(a) \circ & \left.F_{1} \circ\left(\pi_{2}^{*}(a)^{-1} \oplus \pi_{1}^{*}(a)\right)^{-1}\right) \circ F_{2} \\
& \circ\left(\pi_{3}^{*}(a) \circ F_{3} \circ \pi_{5}^{*}(a)^{-1}\right. \\
& \left.\bigoplus \pi_{4}^{*}(a)^{-1}\right) .
\end{aligned}
$$

\section{Proof of Lemma (2.2):}

Let TCoA of complex Lie group $G$ on $\left.\operatorname{Hom}\left(\operatorname{Hom}\left(W_{5} \oplus W_{4}\right), W_{3}^{*}\right), \operatorname{Hom}\left(W_{2} \oplus W_{1}, W\right)\right)$ is induced by the representation $\pi: \mathrm{G} \rightarrow$

$\mathrm{GL}\left(\operatorname{Hom}\left(\operatorname{Hom}\left(W_{5} \oplus W_{4}, W_{3}^{*}\right), \operatorname{Hom}\left(W_{2} \oplus\right.\right.\right.$ $\left.\left.W_{1}, W^{*}\right)\right)$

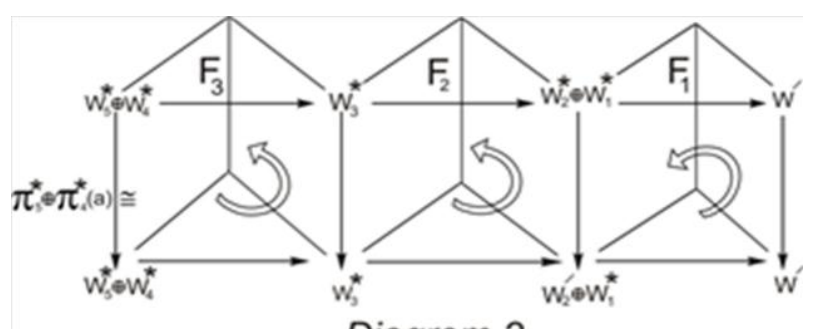

Diagram 2. 
Ahmed.K/Taghreed.H

such that $\pi(a)=\pi(a) \circ F_{1} \circ\left(\pi_{2}(a)^{-1} \bigoplus\right.$ $\left.\left.\pi_{1}(a)\right)^{-1}\right) \circ F_{2} \circ\left(\pi_{3}(a) \circ F_{3} \circ \pi_{5}(a)^{-1} \oplus\right.$ $\left.\pi_{4}(a)^{-1}\right)$

For all $a \in G$. Thus $\pi^{*}$ is a representation from $G$ to Hom - space

This arrow makes the diagram 2 commutative.

\section{Remark (2.4):}

Since $\operatorname{Hom}\left(\operatorname{Hom}\left(W_{5} \oplus W_{4}, W_{3}^{*}\right), \operatorname{Hom}\left(W_{2} \oplus\right.\right.$ $\left.\left.W_{1}, W^{* /}\right)\right) \cong\left(\left(W_{5} \oplus W_{4}, W_{3}^{*}\right)^{*} \otimes\left(\left(W_{2} \oplus W_{1}\right)^{*} \otimes\right.\right.$ $\left.W^{* /}\right) \cong\left(\left(W_{5} \oplus W_{4}\right) \otimes W_{3}\right) \otimes\left(\left(W_{2}^{*} \oplus W_{1}^{*}\right) \otimes W^{* /}\right)$ So we construct an action of $G$ on the product, Let $\pi(G) \rightarrow G L\left(\left(\left(W_{5} \oplus W_{4}\right) \otimes W_{3}\right) \otimes\left(\left(W_{2}^{*} \oplus\right.\right.\right.$ $\left.\left.\left.W_{1}^{*}\right) \otimes W^{* /}\right)\right)$, then $\pi$ forms a representation of $G$ acting on vector space $\left(\left(\left(W_{5} \oplus W_{4}\right) \otimes W_{3}\right) \otimes\left(\left(W_{2}^{*} \oplus W_{1}^{*}\right) \otimes W^{* /}\right)\right)$.

$\left(W_{5}^{*} \times W_{4}^{*}\right) \times W_{3} \times\left(W_{2}^{*} \times W_{1}^{*}\right) \times W^{\prime} \frac{\text { canianical }}{\operatorname{map}}$ $\rightarrow\left(W_{5}^{*} \oplus W_{4}^{*}\right) \otimes W_{3} \otimes\left(W_{2}\right.$ $\left.\bigoplus W^{/}\right)$

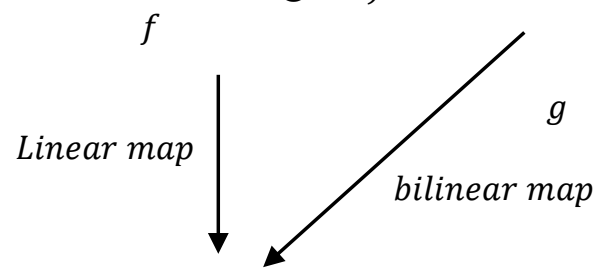

$K \times\left(W_{5} \times W_{4}\right) \times W_{3} \times K \times\left(W_{2} \times W_{1}\right) \times W^{\prime}$

Diagram 3.

$$
\begin{aligned}
f\left(\left(W_{5}^{*} \times W_{4}^{*}\right) \times\right. & \left.W_{3} \times\left(W_{2}^{*} \times W_{1}^{*}\right) \times W^{\prime}\right) \\
= & w^{\prime} \circ\left(w_{2}^{*} \oplus w_{1}^{*}\right) \circ w_{3} \\
& \circ\left(w_{5}^{*} \times w_{4}^{*}\right) \\
g\left(\left(W_{5}^{*} \oplus W_{4}^{*}\right) \otimes\right. & \left.W_{3} \otimes\left(W_{2}^{*} \oplus W_{1}^{*}\right) \otimes W^{\prime}\right) \\
= & w^{\prime} \circ\left(w_{2}^{*} \oplus w_{1}^{*}\right) \circ w_{3} \\
& \circ\left(w_{5}^{*} \times w_{4}^{*}\right)
\end{aligned}
$$

For all $w_{5} \in W_{5}, w_{5} \in W_{5}, w_{4} \in W_{4}, w_{3} \in$ $W_{3}, w_{2} \in W_{2}, w_{1} \in W_{1}, w^{\prime} \in W^{\prime}$.

$G \stackrel{\Delta}{\rightarrow} G \times G \stackrel{\pi_{3} \pi_{2} \pi_{1}}{\longrightarrow} G L\left(W_{5} \oplus W_{4}\right) \times G L\left(W_{3}\right) \times$ $G L\left(W_{2} \oplus W_{1}\right) \times G L\left(W^{\prime}\right)$

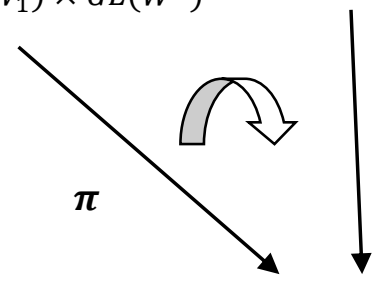

$G L\left(W_{5} \oplus\right.$

$\left.W_{4}\right) \otimes G L\left(W_{3}\right) \otimes G L\left(W_{2} \oplus W_{1}\right) \otimes G L\left(W^{\prime}\right)$

\section{Diagram 4.}

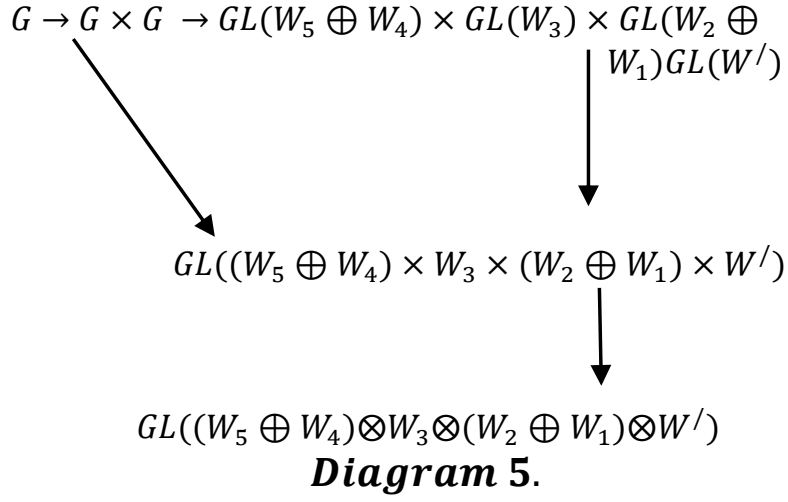

That $\pi$ is a representation of $G$ acting on $G L\left(\left(W_{5} \oplus W_{4}\right) \otimes W_{3} \otimes\left(W_{2} \oplus W_{1}\right) \otimes W^{\prime}\right)$ where $\pi_{i}, i=1,2,3,4,5 \quad$ are five representations of $\mathrm{G}$ acting on $W_{i}, i=1,2,3,4,5$ respectively, thus :

$\pi(a b)=\pi^{\prime}(a b) \circ W^{\prime} \otimes\left(W_{2} \oplus W_{1}\right) \otimes W_{3} \otimes\left(W_{5} \oplus\right.$ $\left.W_{4}\right)$

$\pi^{\prime}(a b) \otimes \pi_{1}^{\prime}(a b) \circ\left(W^{\prime}\right)_{(v)} \circ \pi_{2}(a b)^{-1}\left(W_{2} \oplus\right.$ $\left.W_{1}\right)_{(v)} \circ \pi_{3}(a b) W_{3}(v) \circ \pi_{4}(a b)^{-1}\left(W_{5} \oplus W_{4}\right)_{(v)}=$ $\left.\pi_{4}(a b)^{-1} \oplus \pi_{4}(a b)\right) \otimes W_{3}(a b)^{-1} \otimes \pi_{2}(a b) \otimes$ $\pi_{2}(a b) \otimes \pi_{1}(a b)^{-1} \otimes \pi^{\prime}(a b)$

$\pi(a) \circ \pi(b)=\pi(b)(\pi(a))=\pi(b)\left(\pi^{/}(a) \circ\left(W^{\prime}\right)_{(v)} \circ\right.$ $\pi_{1}(a)\left(W_{2} \oplus W_{1}\right)_{(v)} \circ \pi_{2}(a) W_{3}(v) \circ \pi_{3}(a)\left(W_{5} \oplus\right.$ $\left.W_{4}\right)_{(v)}=\pi^{\prime}(b)\left(\pi^{/}(a) \circ\left(W^{\prime}\right)_{(v)} \circ \pi_{1}(b) \pi_{1}(a)\left(W_{2} \oplus\right.\right.$ $\left.W_{1}\right)_{(v)} \circ \pi_{2}(b) \pi_{2}(a) W_{3}(v) \circ \pi_{3}(b) \pi_{3}(a)\left(W_{5} \oplus\right.$ $\left.W_{4}\right)_{(v)}=\pi^{\prime}(a b) \circ W^{\prime} \otimes\left(W_{2} \oplus W_{1}\right) \otimes W_{3} \otimes\left(W_{5} \oplus\right.$ $\left.W_{4}\right)$.

$$
\pi(a b)=\pi(a) \circ \pi(b)
$$

$\pi$ is a group homomorphism of $G$ on $G L\left(\left(W_{5} \oplus\right.\right.$ $\left.\left.W_{4}\right) \otimes W_{3} \otimes\left(W_{2} \oplus W_{1}\right) \otimes W^{\prime}\right)$.

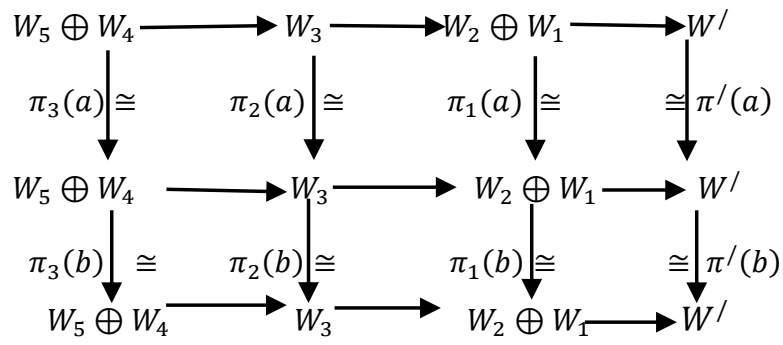

Diagram 6.

\section{3- The TCoA of Complex Lie Groups on Tensor Product \\ We have been introduced the triple Co-action of complex Lie groups by the tensor product of the five representations, which are TCoA-complex Lie groups on tensor product}


$\left(\mathrm{W}_{5}^{*} \otimes\left(\mathrm{W}_{4} \otimes\left(\left(\mathrm{W}_{3}^{*} \otimes \mathrm{W}_{2}\right) \oplus\left(\mathrm{W}_{3}^{*} \otimes \mathrm{W}_{1}\right)\right)\right)\right.$

and constructed this definition. Depending on what has been mentioned above, $\Pi$ is called Triple Co-Action of complex Lie groups on tensor product denoted by (TCoA-complex Lie groups).

\section{Example (3.1):}

Let $\Pi_{1}: \mathbb{R} \longrightarrow \mathrm{GL}(2, \mathbb{C})$ such that $\Pi_{1}(a)=\left(\begin{array}{ll}1 & 0 \\ 1 & 1\end{array}\right)_{2 \times 2}$, for all $a \in \mathbb{R}, \Pi_{2}: \mathbb{R} \longrightarrow$ $\mathrm{GL}(2, \mathbb{C})$ such that $\Pi_{2}(a)=\left(\begin{array}{ll}1 & 2 \\ 0 & 1\end{array}\right)_{2 \times 2}$, for all $a \in$ $\mathbb{R}, \quad \Pi_{3}: \mathbb{R} \longrightarrow \mathrm{GL}(2, \mathbb{C})$ such that $\Pi_{3}(a)=\left(\begin{array}{ll}2 & 0 \\ 2 & 1\end{array}\right)_{2 \times 2}$, for all $a \in \mathbb{R}, \Pi_{4}: \mathbb{R} \longrightarrow$ $\mathrm{GL}(2, \mathbb{C})$ such that $\Pi_{4}(a)=\left(\begin{array}{ll}1 & 0 \\ 1 & 1\end{array}\right)_{2 \times 2}$, for all $a \in$ $\mathbb{R}$ and $\quad \Pi_{5}: \mathbb{R} \longrightarrow \mathrm{GL}(3, \mathbb{C})$ such that $\Pi_{5}(a)=\left(\begin{array}{lll}1 & 0 & 1 \\ 2 & 1 & 0 \\ 0 & 1 & 0\end{array}\right)_{3 \times 3}$, for all $a \in \mathbb{R}$

The representation $\Pi$ of $\mathrm{GL}\left(\mathrm{W}_{5}^{*} \otimes\left(\mathrm{W}_{4} \otimes\left(\left(\mathrm{W}_{3}^{*}\right.\right.\right.\right.$ $\left.\left.\left.\left.\otimes \mathrm{W}_{2}\right) \oplus\left(\mathrm{W}_{3}^{*} \otimes \mathrm{W}_{1}\right)\right)\right)\right)$ is:

$\Pi: \mathrm{G} \longrightarrow \mathrm{GL}\left(\mathrm{W}_{5}^{*} \otimes\left(\mathrm{W}_{4} \otimes\left(\left(\mathrm{W}_{3}^{*} \otimes \mathrm{W}_{2}\right) \oplus\left(\mathrm{W}_{3}^{*}\right.\right.\right.\right.$ $\left.\left.\left.\left.\otimes \mathrm{W}_{1}\right)\right)\right)\right) \cong \mathrm{GL}(\mathrm{M}(8 \times 3), \mathbb{C})$, such that

$\Pi(a)=\left(\left(\left(\Pi_{1}(a) \otimes \Pi_{3}^{*}(a)^{-}{ }^{1} \oplus \Pi_{2}(a) \otimes \Pi_{3}^{*}(a)^{-}\right.\right.\right.$ $\left.\left.\left.{ }^{1}\right) \otimes \Pi_{4}(a)\right) \otimes \Pi_{5}^{*}(a)^{-}{ }^{1}\right) \quad$, where $\quad \Pi^{*} \quad$ is dual representation

$$
=\left(\left[\left(\begin{array}{ll}
1 & 0 \\
1 & 1
\end{array}\right) \otimes\left(\begin{array}{cc}
1 & -2 \\
0 & 2
\end{array}\right) \oplus\left(\begin{array}{cc}
1 & 2 \\
0 & 1
\end{array}\right)\left(\begin{array}{cc}
1 & -2 \\
0 & 2
\end{array}\right)\right] \otimes\left(\begin{array}{cc}
1 & 0 \\
1 & 1
\end{array}\right)\right) \otimes\left(\begin{array}{ccc}
0 & 0 & 1 \\
-\frac{1}{2} & 0 & -\frac{1}{2} \\
-\frac{1}{2} & 1 & \frac{1}{2}
\end{array}\right)
$$

$$
=\left[\left(\begin{array}{cccc}
2 & 2 & -4 & -4 \\
1 & 2 & -2 & -4 \\
0 & 0 & 4 & 4 \\
0 & 0 & 2 & 4
\end{array}\right) \otimes\left(\begin{array}{cc}
1 & 0 \\
1 & 1
\end{array}\right)_{2 \times 2}\right] \otimes\left(\begin{array}{ccc}
0 & 0 & 1 \\
-\frac{1}{2} & 0 & -\frac{1}{2} \\
-\frac{1}{2} & 1 & \frac{1}{2}
\end{array}\right)_{3 \times 3}
$$

$$
=\left(\begin{array}{cccccccc}
2 & 2 & -4 & -4 & 0 & 0 & 0 & 0 \\
1 & 2 & -2 & -4 & 0 & 0 & 0 & 0 \\
0 & 0 & 4 & 4 & 0 & 0 & 0 & 0 \\
0 & 0 & 2 & 4 & 0 & 0 & 0 & 0 \\
2 & 2 & -4 & -4 & 2 & 2 & -4 & -4 \\
1 & 2 & -2 & -4 & 1 & 2 & -2 & -4 \\
0 & 0 & 4 & 4 & 0 & 0 & 4 & 4 \\
0 & 0 & 2 & 4 & 0 & 0 & 2 & 4
\end{array}\right)_{8 \times 8} \otimes\left(\begin{array}{ccc}
0 & 0 & 1 \\
-\frac{1}{2} & 0 & -\frac{1}{2} \\
-\frac{1}{2} & 1 & \frac{1}{2}
\end{array}\right)_{3 \times 3}
$$

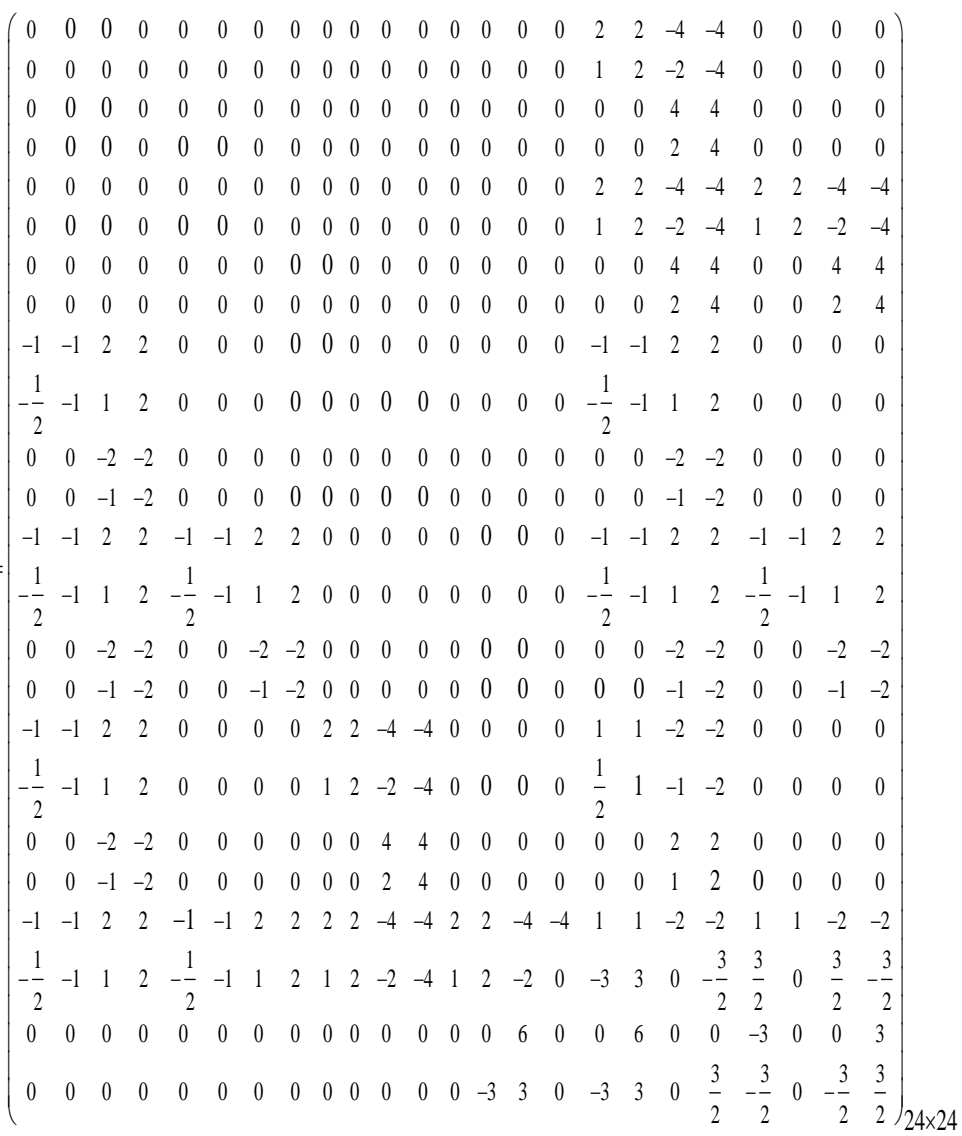

\section{Proposition (3.2):}

Let $\Pi_{\mathrm{i}}: \mathrm{G} \longrightarrow \mathrm{GL}(W i), \Pi_{\mathrm{i}}^{*}: \mathrm{G} \longrightarrow \mathrm{GL}\left(\mathrm{W}_{\mathrm{i}}^{*}\right)$

for $i=1,2,3,4,5$ and the TCoA-complex Lie groups of $G$ on $\left(\mathrm{W}_{5}^{*} \otimes\left(\mathrm{W}_{4} \otimes\left(\left(\mathrm{W}_{3}^{*} \otimes \mathrm{W}_{2}\right) \oplus\left(\mathrm{W}_{3}^{*} \otimes \mathrm{W}_{1}\right)\right)\right)\right)$ is given by a representation $\Pi$ such that $\Pi(a)=\left[\left(\Pi_{1}(a) \circ \mathrm{W}_{1} \circ \mathrm{W}_{3}^{*} \circ \Pi_{3}^{*}\left(a^{-1}\right)\right) \oplus\left(\Pi_{2}(a) \circ \mathrm{W}_{2} \circ\right.\right.$ $\left.\mathrm{W}_{3}^{*} \circ \Pi_{3}^{*}(a)^{-1}\right) \circ \Pi_{4}(a) \circ \mathrm{W}_{4} \circ \mathrm{W}_{5}^{*} \circ \Pi_{5}^{*}(a)^{-1}$, for all $a \in G$. 
Then the TCoA-complex Lie group of $\mathrm{G}$ on $\left(\mathrm{W}_{5}^{*}\right.$ $\left.\otimes\left(\mathrm{W}_{4} \otimes\left(\left(\mathrm{W}_{3}^{*} \otimes \mathrm{W}_{2}\right) \oplus\left(\mathrm{W}_{3}^{*} \otimes \mathrm{W}_{1}\right)\right)\right)\right)$ is also given by a representation $\Pi^{*}$, such that:

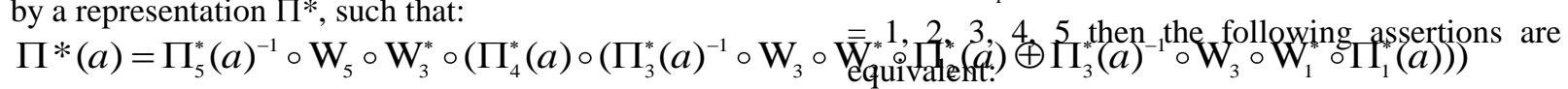
for all $a \in \mathrm{G}$.

Proof: Let TCoA-complex Lie group G on $\left(\mathrm{W}_{5}^{*}\right.$ $\left.\otimes\left(\mathrm{W}_{4} \otimes\left(\left(\mathrm{W}_{3}^{*} \otimes \mathrm{W}_{2}\right) \oplus\left(\mathrm{W}_{3}^{*} \otimes \mathrm{W}_{1}\right)\right)\right)\right)$ is induced by the representation $\Pi: \mathrm{G} \longrightarrow \mathrm{GL}\left(\left(\mathrm{W}_{5}^{*} \otimes\left(\mathrm{W}_{4} \otimes\right.\right.\right.$ $\left.\left.\left(\left(\mathrm{W}_{3}^{*} \otimes \mathrm{W}_{2}\right) \oplus\left(\mathrm{W}_{3}^{*} \otimes \mathrm{W}_{1}\right)\right)\right)\right)$ such that $\Pi(a)=\left(\left(\left(\Pi_{1}(a) \circ \mathrm{W}_{1} \circ \mathrm{W}_{3}^{*} \circ \Pi_{3}\left(a^{-1}\right)\right) \oplus\left(\Pi_{2}(a) \circ \mathrm{W}_{2} \circ\right.\right.\right.$ $\left.\left.\mathrm{W}_{3}^{*} \circ \prod_{3}(a)^{-1}\right) \circ \Pi_{4}(a) \circ \mathrm{W}_{4} \circ \mathrm{W}_{5}^{*} \circ \Pi_{5}(a)^{-1}\right)$, for all $a \in \mathrm{G}, \quad \mathrm{W}_{3}^{\prime} \times \mathrm{W}_{1}^{\prime} \in \mathrm{W}_{3} \times \mathrm{W}_{1}, \quad \Pi_{3} \quad \in$ $\left(\mathrm{W}_{3},\left(\mathrm{~W}_{2} \times \mathrm{W}_{1}\right)\right), \Pi_{4} \in \mathrm{W}_{5} \times \mathrm{W}_{4}$.

To show that $\Pi^{*}: \mathrm{G} \longrightarrow \mathrm{GL}\left(\mathrm{W}_{5}^{*} \otimes\left(\mathrm{W}_{4} \otimes\left(\left(\mathrm{W}_{3}^{*}\right.\right.\right.\right.$ $\left.\left.\left.\left.\otimes \mathrm{W}_{2}\right) \oplus\left(\mathrm{W}_{3}^{*} \otimes \mathrm{W}_{1}\right)\right)\right)\right)^{*}$ is a representation, such that $\Pi^{*}(a)=\left(\left(\left(\Pi_{5}^{*}(a)^{-1} \circ \mathrm{W}_{5} \circ \mathrm{W}_{4}^{*} \circ\left(\Pi_{4}^{*}(a) \circ\left(\Pi_{3}^{*}(a)^{-}\right.\right.\right.\right.\right.$ $1 \circ \mathrm{W}_{3} \circ \mathrm{W}_{2}^{*} \circ \Pi_{2}^{*}(a) \oplus \Pi_{3}^{*}(a)^{-1} \circ$

$$
\left.\left.\mathrm{W}_{3} \circ \mathrm{W}_{1}^{*} \circ \Pi_{1}^{*}(a)\right)\right)
$$

is a representation for all $a \in \mathrm{G}$ and $\Pi_{4}^{*} \in\left(\mathrm{W}_{5}^{*}\right.$ $\left.\otimes \mathrm{W}_{4}\right)^{*}, \Pi_{3}^{*} \in\left(\mathrm{W}_{3},\left(\mathrm{~W}_{2} \otimes \mathrm{W}_{1}\right)\right)^{*}$,

$\left.\Pi_{2}^{*} \times \Pi_{1}^{*} \in\left(\mathrm{W}_{2} \otimes \mathrm{W}_{1}\right)\right)^{*}$ since $\Pi^{*}(a)=\Pi_{5}(a)^{-1} \circ \mathrm{W}_{5} \circ \mathrm{W}_{4}^{*} \circ\left(\Pi_{4}(a) \circ\left(\left(\Pi_{3}(a)\right.\right.\right.$ For all $a \in \mathrm{G}, \quad \Pi_{4}^{*}: \mathrm{W}_{4}^{*} \longrightarrow \mathrm{W}_{5}^{*} \quad$ and $\Pi *(a b)=(\Pi(a b)) *=(\Pi(b) \circ \Pi(a)) *=$ $\Pi^{*}(a) \circ \Pi^{*}(b)$. Thus $\Pi^{*}$ is a representation from $G$ ( $\Pi^{*}$ is a group homoorphism of $G$ )

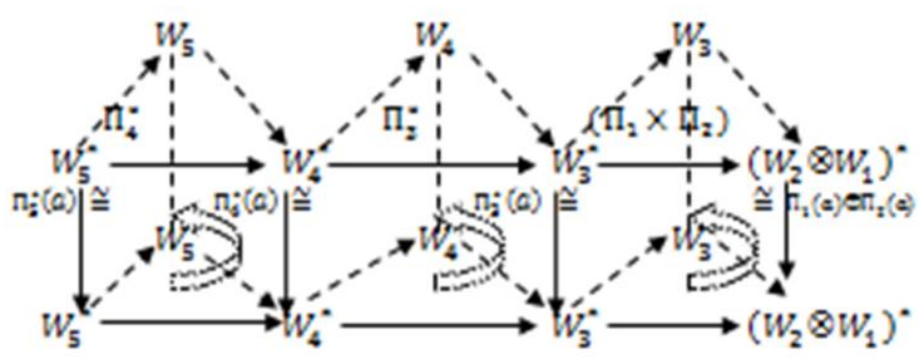

Diagram 7.

This arrow makes the diagram 7 commutative. $\left(\mathrm{W}_{1}^{*}, \mathrm{~W}_{3}^{* *}\right), \mathrm{W}_{5}^{* *} \cong \mathrm{W}_{5}$ and $\mathrm{W}_{3}^{* *} \cong \mathrm{W}_{3}$.

\section{Proposition (3.3):}

Let $W i$ for $\mathrm{i}=1,2,3,4,5$ are finite vector spaces, $\mathrm{W}_{\mathrm{i}}^{*}$ is the dual of vectors $\mathrm{W}_{\mathrm{i}}$, for

(1) $\left[\left(\mathrm{W}_{5}^{*} \otimes\left(\mathrm{W}_{4} \otimes\left(\left(\mathrm{W}_{3}^{*} \otimes \mathrm{W}_{2}\right) \oplus\left(\mathrm{W}_{3}^{*} \otimes \mathrm{W}_{1}\right)\right)\right)\right]^{*}\right.$.

(2) $\quad\left(\left(\left(\mathrm{W}_{3}^{*} \otimes \mathrm{W}_{2}\right)^{* \oplus} \quad\left(\mathrm{W}_{3}^{*} \otimes \mathrm{W}_{1}\right)^{*}\right) \otimes \mathrm{W}_{4}^{*}\right)^{* \otimes}$ $\left.\mathrm{W}_{5}^{* *}\right)$.

(3) $\left.\left.\left(\mathrm{W}_{2}^{*} \otimes \mathrm{W}_{3}\right) \oplus\left(\mathrm{W}_{1}^{*} \otimes \mathrm{W}_{3}\right) \otimes \mathrm{W}_{4}^{*}\right) \otimes \mathrm{W}_{5}\right)$.

(4) $\left.\left(\left(\left(\mathrm{W}_{2}^{*} \otimes \mathrm{W}_{1}^{*}\right) \otimes \mathrm{W}_{3}\right) \otimes \mathrm{W}_{4}^{*}\right) \otimes \mathrm{W}_{5}\right)$.

(5) $\left.\left(\left(\mathrm{W}_{2}^{*} \otimes \mathrm{W}_{1}^{*}\right) \otimes\left(\mathrm{W}_{3}^{*}, \mathrm{~K}\right) \otimes \mathrm{W}_{4}^{*}\right) \otimes \mathrm{W}_{5}\right)$.

(6) $\left.\left.\left(\left(\mathrm{W}_{2}^{*} \otimes \mathrm{W}_{1}^{*}\right) \otimes \mathrm{W}_{3}\right) \otimes \mathrm{W}_{4}^{*}\right) \otimes\left(\mathrm{W}_{5}^{* *}, \mathrm{~K}\right)\right)$.

(7) $\left(\left(\mathrm{W}_{1} \otimes \mathrm{W}_{2}\right) * \otimes \mathrm{W}_{4}^{*}\right) \otimes\left(\mathrm{W}_{3}, \mathrm{~K}\right) \otimes$

$\left.\left.\left.\mathrm{W}_{4}^{*}\right)\right) \otimes \mathrm{W}_{5}\right)$.

(8) $\left[\left(\mathrm{W}_{5} \otimes\left(\mathrm{W}_{4} \otimes\left(\mathrm{W}_{3} \otimes \mathrm{W}_{2}\right) \oplus\left(\mathrm{W}_{3} \otimes \mathrm{W}_{1}\right)\right)\right]^{* * *}\right.$

$= \begin{cases}\left(\mathrm{W}_{5} \otimes\left(\mathrm{W}_{4} \otimes\left(\mathrm{W}_{3} \otimes \mathrm{W}_{2}\right) \oplus\left(\mathrm{W}_{3} \otimes \mathrm{W}_{1}\right)\right)\right. & \text { if } \mathrm{n} \text { is an even number } \\ \left.\left(\mathrm{W}_{3} \otimes \mathrm{W}_{2}\right) * \oplus\left(\mathrm{W}_{3} \otimes \mathrm{W}_{1}\right) * \otimes \mathrm{W}_{4}^{*}\right) \otimes \mathrm{W}_{5} & \text { if } \mathrm{n} \text { is an odd number }\end{cases}$

Proof:

$(1) \cong(2)$ To show $\left[\left(\mathrm{W}_{5}^{*} \otimes\left(\mathrm{W}_{4} \otimes\left(\left(\mathrm{W}_{3}^{*}\right.\right.\right.\right.\right.$

$\left.\left.\left.\otimes \mathrm{W}_{2}\right) \oplus\left(\mathrm{W}_{3}^{*} \otimes \mathrm{W}_{1}\right)\right)\right]^{*} \cong$

$\left.\left(\left(\left(\mathrm{W}_{3}^{*} \otimes \mathrm{W}_{2}\right)^{* \oplus}\left(\mathrm{W}_{3}^{*} \otimes \mathrm{W}_{1}\right)^{*}\right) \otimes \mathrm{W}_{4}^{*}\right)^{* \otimes} \mathrm{W}_{5}^{* *}\right)$.

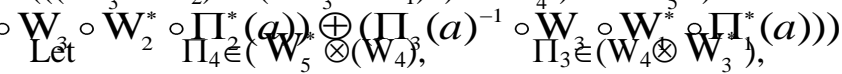

$\Pi_{2} \times \Pi_{1} \in\left(\mathrm{W}_{3}^{*} \otimes\left(\mathrm{W}_{2} \otimes \mathrm{W}_{1}\right)\right), \quad \Pi_{4}^{*} \in\left(\mathrm{W}_{4}^{*} \otimes \mathrm{W}_{5}\right)$,

$\Pi_{3}^{*} \in\left(\mathrm{W}_{3}, \mathrm{~W}_{4}^{*}\right), \Pi_{1}^{*} \times \Pi_{2}^{*}=\left(\Pi_{2} \times \Pi_{1}\right)^{*} \in\left(\left(\mathrm{W}_{1}^{*} \otimes \mathrm{W}_{2}^{*}\right) \otimes \mathrm{W}_{3}\right)$ and there exists an intertwining map $\psi:\left(\mathrm{W}_{5}^{*} \otimes\left(\mathrm{W}_{4} \otimes\left(\left(\mathrm{W}_{3}^{*} \otimes \mathrm{W}_{2}\right) \oplus\left(\mathrm{W}_{3}^{*} \otimes \mathrm{W}_{1}\right)\right)\right)\right) \longrightarrow$ $\left.\left(\left(\left(\mathrm{W}_{3}^{*} \otimes \mathrm{W}_{2}\right)^{* \oplus(} \quad \mathrm{W}_{3}^{*} \otimes \mathrm{W}_{1}\right)^{*}\right) \otimes \quad \mathrm{W}_{4}^{*}\right)^{* \otimes} \mathrm{W}_{5}^{* *}$, such that

$\psi\left(\Pi^{*}(a)\right)(\mathrm{v})=\Pi^{*}(a) \psi(\mathrm{v})$, for all $\mathrm{v} \in \mathrm{W}_{1}^{*} \times \mathrm{W}_{2}^{*}$ and $\psi$ is an invertible map.

$$
\begin{aligned}
& \text { (1) } \cong(3) \text { To show }\left(\mathrm { W } _ { 5 } ^ { * } \otimes \left(\mathrm { W } _ { 4 } \otimes \left(\left(\mathrm{W}_{3}^{*} \otimes \mathrm{W}_{2}\right) \oplus\right.\right.\right. \\
& \text { (2) } \left.\left.\left(\mathrm{W}_{3}^{*} \otimes \mathrm{W}_{1}\right)\right)\right)^{*} \cong
\end{aligned}
$$

$\left.\left(\left(\mathrm{W}_{2}^{*} \otimes \mathrm{W}_{3}^{*}\right) \oplus\left(\mathrm{W}_{1}^{*} \otimes \mathrm{W}_{3}^{*}\right) \otimes \mathrm{W}_{4}^{*}\right) \otimes \mathrm{W}_{5}\right)$, since

$\left(\mathrm{W}_{3}^{*}, \mathrm{~W}_{2}\right)^{*} \cong\left(\mathrm{W}_{2}^{*}, \mathrm{~W}_{3}^{* *}\right), \quad\left(\mathrm{W}_{3}^{*}, \mathrm{~W}_{1}\right)^{*} \cong$

By the same methods, we have the other parts. 
Ahmed .K/Taghreed.H

Example (3.4):

Let $\Pi_{\mathrm{i}}, \mathrm{i}=1,2,3,4, \Pi_{\mathrm{i}}: \mathrm{S}^{1} \longrightarrow \mathrm{SO}(2) \subset$ $\mathrm{GL}(2, \mathbb{C})$ and $\Pi_{5}: \mathrm{S}^{1} \longrightarrow \mathrm{O}(3) \subset \mathrm{GL}(3, \mathbb{C})$, where $\mathrm{G}$ $=\mathrm{S}^{1},(\mathrm{n}=2, \mathrm{~m}=3)$ and $W i, \mathrm{i}=1,2,3,4$ are the $\mathbb{C}$ vector spaces of dimensional 2 and $\mathrm{W}_{5}$ is the $\mathbb{C}$ vector space of dimensional 3 such that,

$\Pi_{1}\left(\mathrm{e}^{\mathrm{i} \theta}\right)=\Pi_{2}\left(\mathrm{e}^{\mathrm{i} \theta}\right)=\Pi_{3}\left(\mathrm{e}^{\mathrm{i} \theta}\right)=\Pi_{4}\left(\mathrm{e}^{\mathrm{i} \theta}\right)=\left(\begin{array}{cc}\cos \theta & -\sin \theta \\ \sin \theta & \cos \theta\end{array}\right)$ $, 0 \leq \theta \leq 2 \pi, \mathrm{i}^{2}=-1, \Pi_{5}\left(\mathrm{e}^{\mathrm{i} \theta}\right)=\left(\begin{array}{ccc}1 & 0 & 0 \\ 0 & \cos \theta & -\sin \theta \\ 0 & \sin \theta & \cos \theta\end{array}\right), 0$

$\leq \theta \leq 2 \pi$. The TCoA-complex Lie group $\mathrm{G}$ on $\left(\mathrm{W}_{5}^{*}\right.$ $\otimes\left(\mathrm{W}_{4} \otimes\left(\left(\mathrm{W}_{3}^{*} \otimes \mathrm{W}_{2}\right) \oplus\left(\mathrm{W}_{3}^{*} \otimes \mathrm{W}_{1}\right)\right)\right)$ is a representation:

П: $\mathrm{S}^{1} \longrightarrow \mathrm{GL}\left(\mathrm{W}_{5}^{*} \otimes\left(\mathrm{W}_{4} \otimes\left(\left(\mathrm{W}_{3}^{*} \otimes \mathrm{W}_{2}\right) \oplus\left(\mathrm{W}_{3}^{*}\right.\right.\right.\right.$ $\left.\left.\otimes \mathrm{W}_{1}\right)\right)$ ) such that

$\Pi^{*}(a)=\left(\Pi_{5}(a)^{-1} \circ \mathrm{W}_{5} \circ \mathrm{W}_{4}^{*} \circ\left(\Pi_{4}^{*}(a) \circ\left(\left(\Pi_{3}(a)^{-1} \circ \mathrm{W}_{3} \circ\right.\right.\right.\right.$

$\Pi^{*}\left(\mathrm{e}^{\mathrm{i} \theta}\right)=\Pi_{5}\left(\mathrm{e}^{-\mathrm{i} \theta}\right) \otimes\left(\Pi_{4}^{*}\left(\mathrm{e}^{\mathrm{i} \theta}\right) \otimes\left(\left(\Pi_{3}\left(\mathrm{e}^{-\mathrm{i} \theta}\right) \otimes \Pi_{2}^{*}\left(\mathrm{e}^{\mathrm{i} \theta}\right)\right) \oplus_{2}\left(\Pi_{3}\left(\mathrm{e}^{-\mathrm{i} \theta}\right) \otimes \Pi_{* 4}^{*}\left(\mathrm{e}^{\mathrm{i} \theta}\right)\right)\right.\right.$

$=\left(\begin{array}{ccc}1 & 0 & 0 \\ 0 & \cos \theta & -\sin \theta \\ 0 & \sin \theta & \cos \theta\end{array}\right) \otimes\left(\begin{array}{cc}\cos \theta & -\sin \theta \\ \sin \theta & \cos \theta\end{array}\right) \otimes\left(\left(\begin{array}{cc}\cos \theta & \sin \theta \\ -\sin \theta & \cos \theta\end{array}\right) \otimes\left(\begin{array}{cc}\cos \theta & -\sin \theta \\ \sin \theta & \cos \theta\end{array}\right)\right) \oplus$

$\left(\left(\begin{array}{cc}\cos \theta & \sin \theta \\ -\sin \theta & \cos \theta\end{array}\right) \otimes\left(\begin{array}{cc}\cos \theta & -\sin \theta \\ \sin \theta & \cos \theta\end{array}\right)\right)$

$=\left(\begin{array}{cccccc}\cos \theta & 0 & 0 & -\sin \theta & 0 & 0 \\ 0 & \cos ^{2} \theta & -\sin \theta \cos \theta & 0 & -\sin \theta \cos \theta & \sin ^{2} \theta \\ 0 & \sin \theta \cos \theta & \cos ^{2} \theta & 0 & -\sin ^{2} \theta & -\sin \theta \cos \theta \\ \sin \theta & 0 & 0 & \cos \theta & 0 & 0 \\ 0 & \sin \theta \cos \theta & -\sin ^{2} \theta & 0 & \cos ^{2} \theta & -\sin \theta \cos \theta \\ 0 & \sin ^{2} \theta & \sin \theta \cos \theta & 0 & \sin \theta \cos \theta & \cos ^{2} \theta\end{array}\right)_{6 \times 6}$

$\left[\begin{array}{cccc}\cos ^{2} \theta & \sin \theta \cos \theta & -\sin \theta \cos \theta & -\sin ^{2} \theta \\ -\sin \theta \cos \theta & \cos ^{2} \theta & \sin ^{2} \theta & -\sin \theta \cos \theta \\ \sin \theta \cos \theta & \sin ^{2} \theta & \cos ^{2} \theta & \sin \theta \cos \theta \\ -\sin ^{2} \theta & \sin \theta \cos \theta & -\sin \theta \cos \theta & \cos ^{2} \theta\end{array}\right)_{4 \times 4}$

$\left(\begin{array}{cccc}\cos ^{2} \theta & \sin \theta \cos \theta & -\sin \theta \cos \theta & -\sin ^{2} \theta \\ -\sin \theta \cos \theta & \cos ^{2} \theta & \sin ^{2} \theta & -\sin \theta \cos \theta \\ \sin \theta \cos \theta & \sin ^{2} \theta & \cos ^{2} \theta & \sin \theta \cos \theta \\ -\sin ^{2} \theta & \sin \theta \cos \theta & -\sin \theta \cos \theta & \cos ^{2} \theta\end{array}\right)_{4 \times 4}$

$=\left(\begin{array}{cccccc}\cos \theta & 0 & 0 & -\sin \theta & 0 & 0 \\ 0 & \cos ^{2} \theta & -\sin \theta \cos \theta & 0 & -\sin \theta \cos \theta & \sin ^{2} \theta \\ 0 & \sin \theta \cos \theta & \cos ^{2} \theta & 0 & -\sin ^{2} \theta & -\sin \theta \cos \theta \\ \sin \theta & 0 & 0 & \cos \theta & 0 & 0 \\ 0 & \sin \theta \cos \theta & -\sin ^{2} \theta & 0 & \cos ^{2} \theta & -\sin \theta \cos \theta \\ 0 & \sin ^{2} \theta & \sin \theta \cos \theta & 0 & \sin \theta \cos \theta & \cos ^{2} \theta\end{array}\right)_{6 \times 6}$

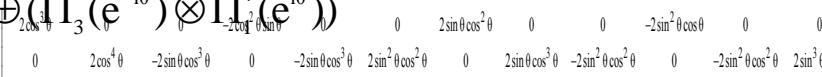
$=2 \sin \theta \cos ^{3} \theta \quad 2 \cos ^{4} \theta \quad 0 \quad-2 \sin ^{2} \theta \cos ^{2} \theta-2 \sin ^{3} \cos ^{3} \theta \quad 0 \quad 2 \sin ^{2} \cos ^{2} \theta-2 \sin ^{3} \cos ^{3} \theta \quad 0 \quad-2 \sin ^{3} \theta \cos ^{3} \theta-2 \sin ^{2} \theta \cos ^{2} \theta$

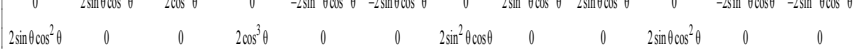

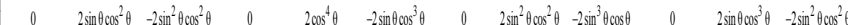
$\begin{array}{llllllll}0 & 2 \sin ^{2} \theta \cos ^{2} \theta & 2 \sin \theta \cos ^{3} \theta \quad 0 \quad 2 \sin ^{3} \theta \cos ^{3} \theta & 2 \cos ^{4} \theta \quad 0 & 2 \sin ^{3} \theta \cos ^{3} \theta & 2 \sin ^{2} \theta \cos ^{2} \theta \quad 0 \quad 2 \sin ^{2} \theta \cos ^{2} \theta & 2 \sin \theta \cos ^{3} \theta\end{array}$

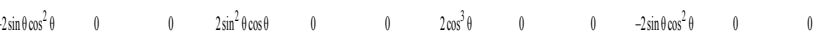
$\begin{array}{lllllll}-2 \sin ^{2} \cos ^{3} \theta & 2 \sin ^{2} \theta \cos ^{2} \theta \quad 0 \quad 2 \sin ^{2} \theta \cos ^{2} \theta & -2 \sin ^{3} \theta \cos \theta \quad 0 \quad 2 \cos ^{4} \theta & -2 \sin ^{2} \theta \cos ^{3} \theta \quad 0 & -2 \sin ^{3} \theta \cos ^{3} \theta & 2 \sin ^{2} \theta \cos ^{2} \theta\end{array}$ $\begin{array}{llllllll}0 & -2 \sin ^{2} \theta \cos ^{2} \theta & -2 \sin \theta \cos ^{3} \theta \quad 0 & -2 \sin ^{3} \theta \cos ^{3} \theta & 2 \sin ^{2} \theta \cos ^{2} \theta \quad 0 \quad 2 \sin ^{2} \cos ^{3} \theta & 2 \cos ^{4} \theta \quad 0 & -2 \sin ^{2} \theta \cos ^{2} \theta & -2 \sin ^{2} \theta \cos ^{3} \theta\end{array}$

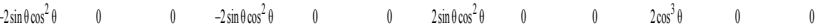

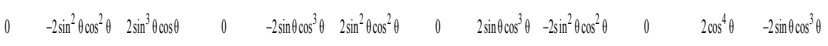
$\begin{array}{llllllll}0 & -2 \sin ^{3} \theta \cos \theta & -2 \sin ^{2} \theta \cos ^{2} \theta \quad 0 & -2 \sin ^{2} \theta \cos ^{2} \theta & -2 \sin \theta \cos ^{3} \theta \quad 0 & 2 \sin ^{2} \theta \cos ^{2} \theta & 2 \sin ^{3} \theta \cos ^{3} \theta \quad 0 \quad 2 \sin ^{3} \cos ^{3} \theta \quad 2 \cos ^{4} \theta\end{array}$

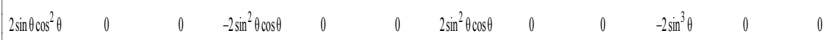
$\begin{array}{lllllllll}0 & -2 \sin ^{2} \cos ^{3} \theta & -2 \sin ^{2} \theta \cos ^{2} \theta \quad 0 & -2 \sin ^{2} \theta \cos ^{2} \theta & 2 \sin ^{3} \theta \cos \theta \quad 0 \quad 2 \sin ^{2} \theta \cos ^{2} \theta & -2 \sin ^{3} \theta \cos \theta \quad 0 & -2 \sin ^{3} \theta \cos \theta & 2 \sin ^{4} \theta\end{array}$ $2 \sin ^{2} \theta \cos ^{2} \theta \quad 2 \sin ^{2} \cos ^{3} \theta \quad 0 \quad-2 \sin ^{3} \theta \cos \theta \quad-2 \sin ^{2} \theta \cos ^{2} \theta \quad 0 \quad 2 \sin ^{3} \theta \cos \theta \quad 2 \sin ^{2} \theta \cos ^{2} \theta \quad 0 \quad-2 \sin ^{4} \theta \quad-2 \sin ^{3} \theta \cos \theta$

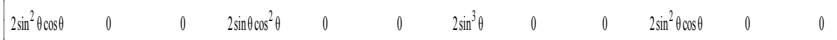

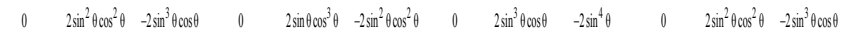

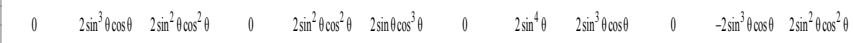

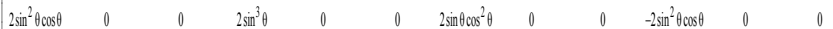

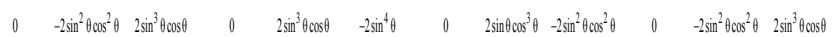

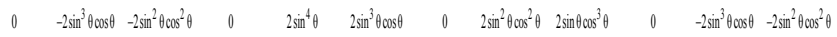

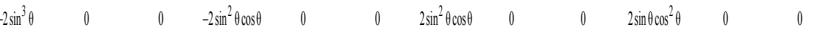

$-2 \sin ^{3} \theta \cos \theta \quad 2 \sin ^{4} \theta \quad 0 \quad-2 \sin ^{2} \theta \cos ^{2} \theta \quad 2 \sin ^{3} \theta \cos \theta \quad 0 \quad 2 \sin ^{2} \theta \cos ^{2} \theta-2 \sin ^{2} \theta \cos \theta \quad 0 \quad 2 \sin ^{2} \cos ^{3} \theta-2 \sin ^{2} \theta \cos ^{2} \theta$ $-2 \sin ^{4} \theta \quad-2 \sin ^{3} \theta \cos \theta \quad 0 \quad-2 \sin ^{3} \theta \cos \theta-2 \sin ^{2} \theta \cos ^{2} \theta \quad 0 \quad 2 \sin ^{3} \theta \cos ^{2} \theta \sin ^{2} \theta \cos ^{2} \theta \quad 0 \quad 2 \sin ^{2} \theta \cos ^{2} \theta \quad 2 \sin ^{2} \theta \cos ^{3} \theta$ 


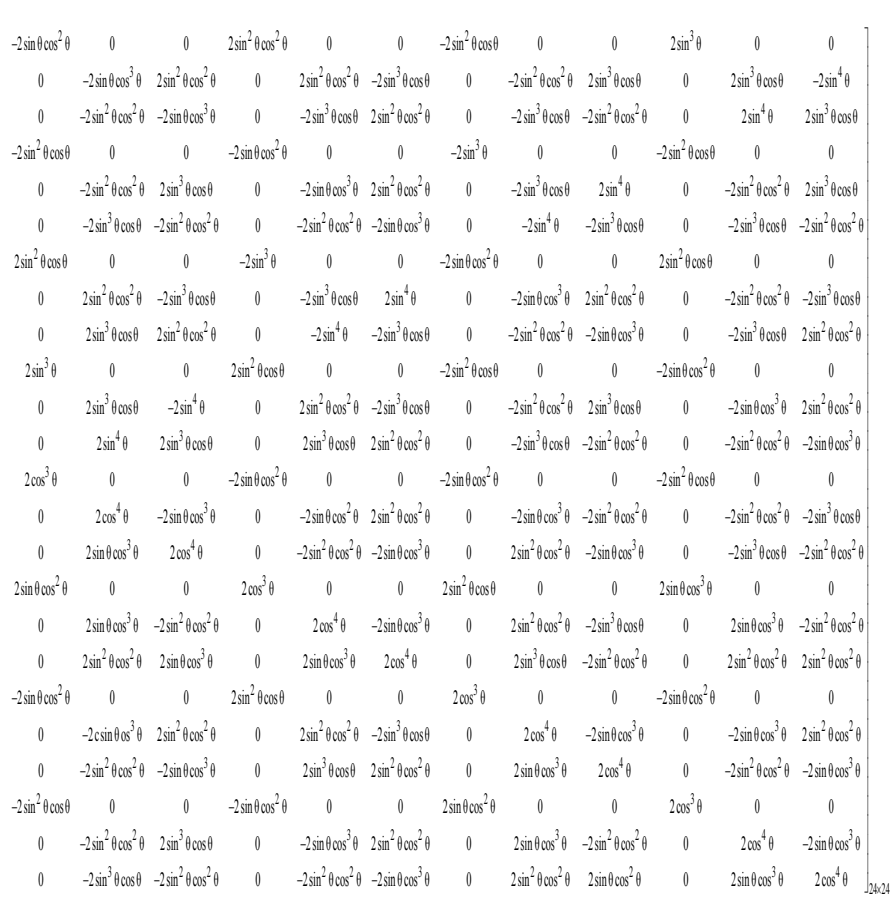

\section{Proposition (3. 5):}

Let $\Pi_{i}, i=1,2,3,4,5$ be representations of $\mathrm{G}$ acting on $\mathrm{K}$-finite dimensional vector spaces $\mathrm{W}_{\mathrm{i}}, \mathrm{i}=$ 1,2,3,4,5 respectively, then the $T C o A$-reductive Lie group of $\mathrm{G}$ on $\underset{\mathrm{K}}{\mathrm{Hom}}$ $\left(\mathrm{W}_{5}, \operatorname{Hom}\left(\mathrm{W}_{4}^{*}, \operatorname{Hom}\left(\left(\mathrm{W}_{3}, \mathrm{~W}_{2}\right) \oplus \operatorname{Hom}\left(\mathrm{W}_{3}, \mathrm{~W}_{1}\right)\right)\right)\right.$ is equivalent to the representation $\Pi_{5}^{*} \otimes\left(\Pi_{4} \otimes\left(\left(\Pi_{3}^{*}\right.\right.\right.$ $\left.\left.\left.\left.\otimes \Pi_{2}\right) \oplus \quad\left(\Pi_{3}^{*} \otimes \Pi_{1}\right)\right)\right)\right) \quad$ of $\quad \mathrm{G} \quad$ on $\quad \mathrm{GL}\left(\mathrm{W}_{5}^{*}\right.$ $\left.\otimes\left(\mathrm{W}_{4} \otimes\left(\left(\mathrm{W}_{3}^{*}, \mathrm{~W}_{2}\right) \oplus\left(\mathrm{W}_{3}^{*}, \mathrm{~W}_{1}\right)\right)\right)\right)$.

\section{Proof: $\quad$ To show that:}

$\psi:\left(\mathrm{W}_{5}^{*} \otimes\left(\mathrm{W}_{4} \otimes\left(\left(\mathrm{W}_{3}^{*} \otimes \mathrm{W}_{2}\right) \oplus\left(\mathrm{W}_{3}^{*} \otimes \mathrm{W}_{1}\right)\right)\right)\right) \longrightarrow$

$\operatorname{Hom}\left(\mathrm{W}_{5}, \operatorname{Hom}\left(\mathrm{W}_{4}^{*}, \operatorname{Hom}\left(\left(\mathrm{W}_{3}, \mathrm{~W}_{2}\right) \oplus\right.\right.\right.$

$\left.\operatorname{Hom}\left(\mathrm{W}_{3}, \mathrm{~W}_{1}\right)\right)$ ) is bilinear map, defined by $\psi\left(\mathrm{W}_{5}^{*}, \mathrm{w}_{1}\right)=\mathrm{F}$ for all $\mathrm{W}_{5}^{*} \in \mathrm{W}_{5}$ and $\mathrm{w}_{1} \in \mathrm{W}_{1}$,

where $\mathrm{F}: \mathrm{W}_{5} \longrightarrow \mathrm{W}_{1}$ is a linear map defined by $\mathrm{F}(\mathrm{v})$ $=\mathrm{W}_{5}^{*}(\mathrm{v}) \mathrm{W}_{1}$, for all $\mathrm{W}_{5}^{*}, \mathrm{~W}_{5}^{* \prime} \in \mathrm{W}_{5}^{*}, \mathrm{v} \in \mathrm{W}_{5}$, $\alpha, \beta \in \mathrm{K}, \mathrm{w}_{1} \in \mathrm{W}_{1}$ $\psi\left(\alpha \mathrm{W}_{5}^{*}+\beta \mathrm{w}_{5}^{*^{\prime}}, \mathrm{w}_{1}\right)=\left(\alpha \mathrm{W}_{5}^{*}+\beta \mathrm{w}_{5}^{*^{\prime}}(\mathrm{v})\right) \mathrm{w}_{1}$

$$
\begin{aligned}
& =\alpha \mathrm{W}_{5}^{*}(\mathrm{v}) \mathrm{w}_{1}+\beta \mathrm{w}_{5}^{*^{\prime}}(\mathrm{v}) \mathrm{w}_{1} \\
& =\alpha \psi\left(\mathrm{w}_{5}^{*}, \mathrm{w}_{1}\right)+\beta \psi\left(\mathrm{w}_{5}^{*^{\prime}}, \mathrm{w}_{1}\right)
\end{aligned}
$$

Other for all $\mathrm{w}_{1}, \mathrm{~W}_{1}^{\prime} \in \mathrm{W}_{1}$ and $\mathrm{W}_{5}^{*} \in \mathrm{W}_{5}^{*}$ $\psi\left(, \alpha \mathrm{w}_{1}+\beta \mathrm{w}_{1}^{\prime}\right)=\left(\mathrm{w}_{5}^{*}(\mathrm{v})\left(\alpha \mathrm{w}_{1}+\beta \mathrm{w}_{1}^{\prime}\right)\right.$
Ahmed.K/Taghreed.H

$$
\begin{gathered}
=\mathrm{w}_{5}^{*}(\mathrm{v})\left(\alpha \mathrm{w}_{1}\right)+\mathrm{W}_{5}^{*^{\prime}}(\mathrm{v})\left(\beta \mathrm{w}_{1}^{\prime}\right) \\
=\alpha \mathrm{W}_{5}^{*}(\mathrm{v}) \mathrm{w}_{1}+\beta \mathrm{w}_{5}^{*^{\prime}}(\mathrm{v}) \mathrm{W}_{1}^{\prime}
\end{gathered}
$$

$\psi\left(\mathrm{W}_{5}^{*}, \alpha \mathrm{w}_{1}+\beta \mathrm{w}_{1}^{\prime}\right)=\alpha \psi\left(\mathrm{W}_{5}^{*}, \mathrm{w}_{1}\right)+\beta \psi\left(\mathrm{W}_{5}^{*}\right.$, $\left.\mathrm{W}_{1}^{\prime}\right)$.

So $\psi$ : $\left.\mathrm{W}_{5}^{*} \times\left(\mathrm{W}_{4} \times\left(\left(\mathrm{W}_{3}^{*} \times \mathrm{W}_{2}\right) \oplus\left(\mathrm{W}_{3}^{*} \times \mathrm{W}_{1}\right)\right)\right)\right) \longrightarrow$ $\underset{\mathrm{K}}{\operatorname{Hom}}\left(\mathrm{W}_{5}, \operatorname{Hom}\left(\mathrm{W}_{4}^{*}, \operatorname{Hom}\left(\left(\mathrm{W}_{3}, \mathrm{~W}_{2}\right)\right.\right.\right.$

$\left.\left.\oplus \operatorname{Hom}\left(\mathrm{W}_{3}, \mathrm{~W}_{1}\right)\right)\right)$ is a bilinear map, thus by using the tensor product and universal property of this tensor product, we get a unique linear map $\phi$.

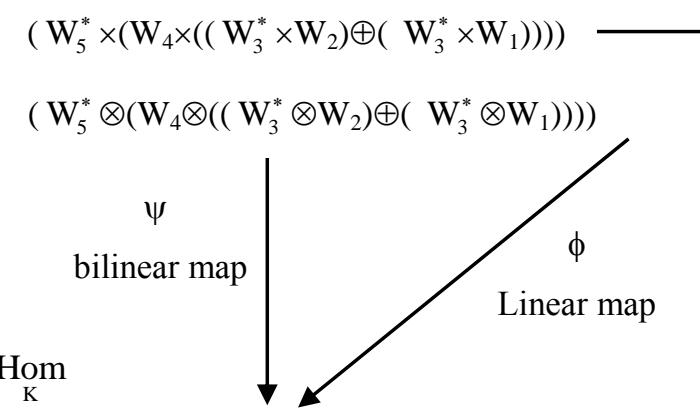

$\left(\mathrm{W}_{5}, \operatorname{Hom}\left(\mathrm{W}_{4}^{*}, \operatorname{Hom}\left(\left(\mathrm{W}_{3}, \mathrm{~W}_{2}\right) \oplus \operatorname{Hom}\left(\mathrm{W}_{3}, \mathrm{~W}_{1}\right)\right)\right)\right.$

\section{Diagram 8.}

So by universal property of tensor product $\mathrm{W}_{5}^{*}$ $\left.\times\left(\mathrm{W}_{4} \times\left(\left(\mathrm{W}_{3}^{*} \times \mathrm{W}_{2}\right) \oplus\left(\mathrm{W}_{3}^{*} \times \mathrm{W}_{1}\right)\right)\right)\right)$ there exists a unique linear map $\phi: \mathrm{W}_{5}^{*} \otimes\left(\mathrm{W}_{4} \otimes\left(\left(\mathrm{W}_{3}^{*}\right.\right.\right.$ $\left.\left.\left.\left.\otimes \mathrm{W}_{2}\right) \oplus\left(\mathrm{W}_{3}^{*} \otimes \mathrm{W}_{1}\right)\right)\right)\right) \longrightarrow \underset{\mathrm{K}}{\operatorname{Hom}}\left(\mathrm{W}_{5}, \operatorname{Hom}\left(\mathrm{W}_{4}^{*}\right.\right.$,

$\left.\operatorname{Hom}\left(\left(\mathrm{W}_{3}, \mathrm{~W}_{2}\right) \oplus \operatorname{Hom}\left(\mathrm{W}_{3}, \mathrm{~W}_{1}\right)\right)\right)$. This makes the above diagram commutative:

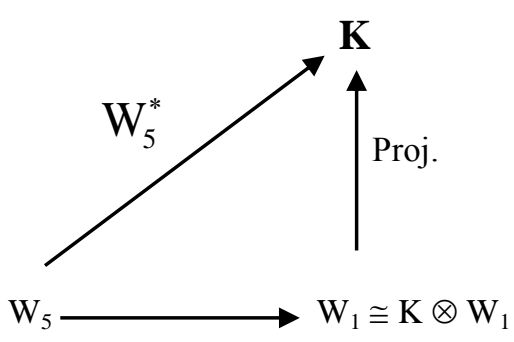

Diagram 9.

Consider the composition of linear maps where $\mathrm{W}_{5}^{*}(\mathrm{v})$ is defined as follows:

$\mathrm{F}(\mathrm{v})=\mathrm{w}_{1}, \exists$ ! $\mathrm{k} \in \mathrm{K}$, such that $\mathrm{w}_{1} \longrightarrow\left(\mathrm{k}, \mathrm{w}_{1}\right)$ since all maps are linear and $\mathrm{k}$ is unique, put $\mathrm{W}_{5}^{*}(\mathrm{v})=\mathrm{k}$ related to $\mathrm{w}_{1}$. 
Define

$\zeta:$

$\underset{\mathrm{K}}{\mathrm{Hom}}$

$\left(\mathrm{W}_{5}, \operatorname{Hom}\left(\mathrm{W}_{4}^{*}, \operatorname{Hom}\left(\left(\mathrm{W}_{3}, \mathrm{~W}_{2}\right) \oplus \operatorname{Hom}\left(\mathrm{W}_{3}, \mathrm{~W}_{1}\right)\right)\right)\right.$

$\longrightarrow \mathrm{W}_{5}^{*} \otimes\left(\mathrm{W}_{4} \otimes\left(\left(\mathrm{W}_{3}^{*} \otimes \mathrm{W}_{2}\right) \oplus \quad\left(\mathrm{W}_{3}^{*} \otimes \mathrm{W}_{1}\right)\right)\right) \quad$ by $\zeta\left(\mathrm{F}^{\prime}\right)=\mathrm{W}_{5}^{*}(\mathrm{v}) \mathrm{W}_{1}$.

Define $\mathrm{W}_{5}^{*}: \mathrm{W}_{5} \longrightarrow \mathrm{K}$ by $\mathrm{W}_{5}^{*}(\mathrm{v})=\mathrm{k}$, where $\mathrm{k}$ is given by $\zeta\left(F^{\prime}(v)\right)=\left(k, F^{\prime}(v)\right)$

We can show that $\mathrm{W}_{5}^{*}$ is linear put $\mathrm{F}^{\prime}(\mathrm{v})=\mathrm{w}_{1}$, for all $\quad \mathrm{F}^{\prime} \in \underset{\mathrm{K}}{\mathrm{Hom}}\left(\mathrm{W}_{5}, \operatorname{Hom}\left(\mathrm{W}_{4}^{*}, \operatorname{Hom}\left(\left(\mathrm{W}_{3}\right.\right.\right.\right.$, $\left.\left.\left.\mathrm{W}_{2}\right) \oplus \operatorname{Hom}\left(\mathrm{W}_{3}, \mathrm{~W}_{1}\right)\right)\right), \mathrm{W}_{1} \in \mathrm{W}_{1}$ and $\mathrm{W}_{5}^{*} \in \mathrm{W}_{5}$ and is related to $\mathrm{W}_{1}$.

$$
\begin{aligned}
\mathrm{F}^{\prime}\left(\alpha \mathrm{v}_{1}+\beta \mathrm{v}_{2}\right) & =\alpha \mathrm{F}^{\prime}\left(\mathrm{v}_{1}\right)+\beta \mathrm{F}^{\prime}\left(\mathrm{v}_{2}\right) \\
& =\alpha \mathrm{k}_{1}+\beta \mathrm{k}_{2} \\
& =\alpha \mathrm{W}_{5}^{*}(\mathrm{v})+\beta \mathrm{W}_{5}^{*}(\mathrm{v}), \text { for all } \mathrm{W}_{5}^{*}
\end{aligned}
$$

$\in \mathrm{W}_{5}$

Where: $\mathrm{W}_{5}^{*}\left(\mathrm{v}_{1}\right)=\mathrm{k}_{1} \Rightarrow \mathrm{W}_{5}^{*}\left(\alpha \mathrm{v}_{1}\right)=\alpha \mathrm{k}_{1}$,

$$
\begin{aligned}
& \mathrm{w}_{5}^{*}\left(\alpha \mathrm{v}_{1}+\beta \mathrm{v}_{2}\right)=\left(\alpha \mathrm{k}_{1}+\beta \mathrm{k}_{2}\right) \\
& \mathrm{w}_{5}^{*}\left(\mathrm{v}_{2}\right)=\mathrm{k}_{2} \Rightarrow \mathrm{w}_{5}^{*}\left(\beta \mathrm{v}_{2}\right)=\beta \mathrm{k}_{2}
\end{aligned}
$$

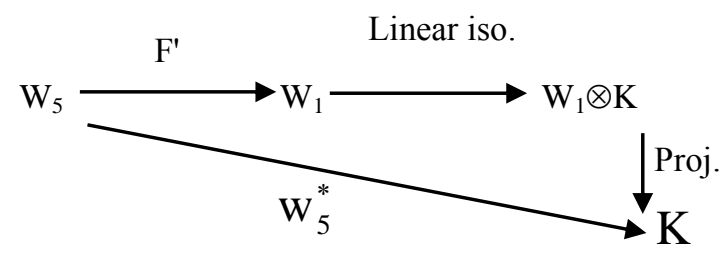

Diagram 10.

Clear $\mathrm{F}^{\prime}$ is a linear and $\zeta^{-1}=\phi$, thus $\zeta$ is linear map. Related between the $T C o A$ of reductive Lie groups of $\mathrm{G}$ on $\underset{\mathrm{K}}{\mathrm{Hom}}\left(\mathrm{W}_{5}, \operatorname{Hom}\left(\mathrm{W}_{4}^{*}, \operatorname{Hom} \quad\left(\left(\mathrm{W}_{3}\right.\right.\right.\right.$, $\left.\left.\left.\mathrm{W}_{2}\right) \oplus \operatorname{Hom}\left(\mathrm{W}_{3}, \mathrm{~W}_{1}\right)\right)\right)$ and $T C o A$ of reductive Lie groups of $\mathrm{G}$ on $\mathrm{W}_{5}^{*} \otimes\left(\mathrm{W}_{4} \otimes\left(\left(\mathrm{W}_{3}^{*} \otimes \mathrm{W}_{2}\right) \oplus\left(\mathrm{W}_{3}^{*}\right.\right.\right.$ $\left.\left.\otimes W_{1}\right)\right)$ ) up to the representation given above:

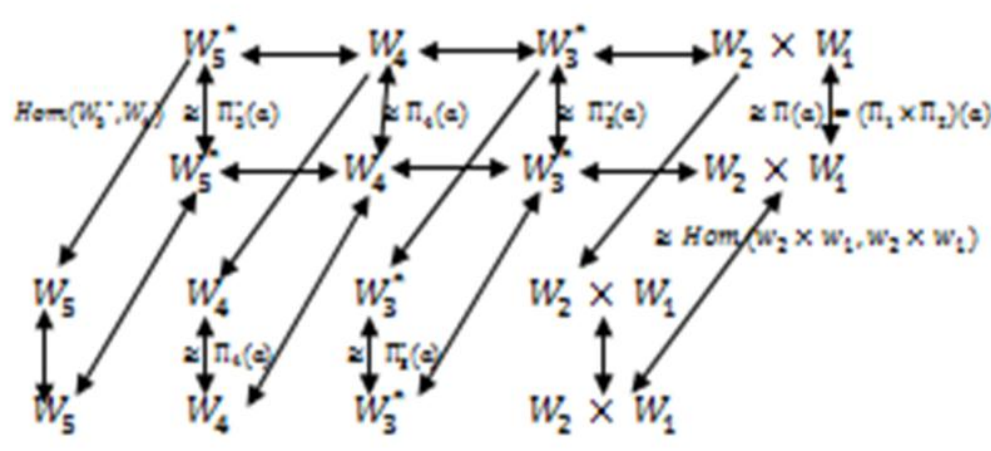

\section{Diagram 11.}

\section{References}

[1] Hussian, M.J. " Local Property of the Lie Algebra and the Exponential Map",M.Sc. Thesis, college of Education, Al - Mustansiriya University, 2010.

[2] Jacobson, N., "Basic Algebra II", Second Edition, Yale University, 2012.

[3] Kac, V., "Introduction to Lie Algebras", Vol. 18.745, Patrick Lam, 2004.

[4] Kirillov, A.Jr., "Introduction to Lie Groups and Lie Algebras",Vol.11794, Sunt at stony Brook, 2004.

[5] Knapp, A.W., "Lie Groups Beyond an Introduction", Progress in Mathematics 140, Brikuauser Boston Inc. Boston, MA, 2002.

[6] Lee, D.H., "The Structure of Complex Lie Groups", New York, ISBN, May, 2001.

[7] Majeed, T.H., "The Universal Property of Tensor Product for Representation of Lie Groups", Ph.D. Thesis, college of Education, Al-Mustansiriya University, 2012.

[8] Nadjafikhah, M. and Chamazkoti, R,. "The Special Linear Representations of Compact Lie Groups", Mathematics Science, Vol.4, No,3, Iran, 2010.

[9] Onishchik, A.L., "Lie Algebra, Reductive", Hazewinkel, Michiel, Encyclopedia of Mathematics, Springer, ISBN 978-1-55608010-4, 2001.

[10] Popov, V.L., "Reductive Group", Hazewinkel, Michiel, Encyclopedia of Mathematics, Springer, ISBN 968-1-55608-010-4, 2001. 


\section{أنواع معينة لقعل زمر لي المركبة}

تغريا حر مجيد المبائ

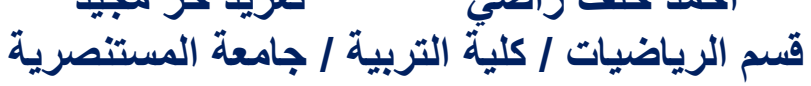

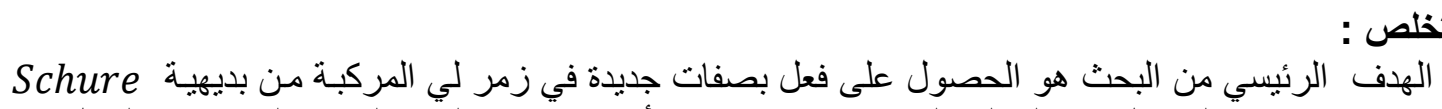

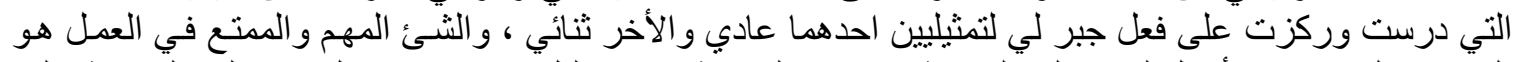

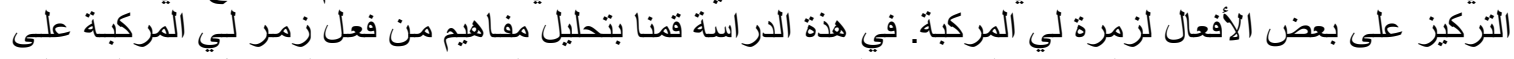

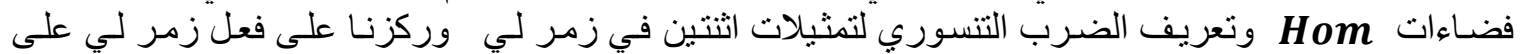
فضاءات Hom، باستخدام التكافؤ

$$
\operatorname{Hom}\left(w_{2}, w_{1}\right) \cong w_{2}^{*} \otimes w_{1}
$$

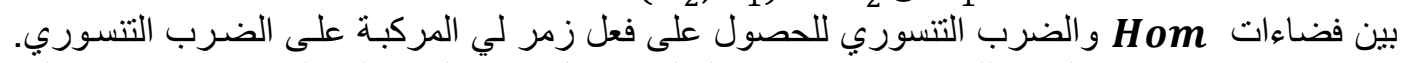

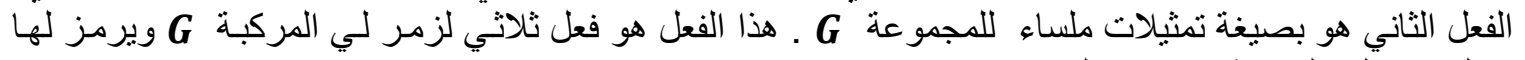

$\operatorname{Hom}\left(\operatorname{Hom}\left(\left(w_{5} \oplus w_{4}\right), w_{3}^{*}\right), \operatorname{Hom}\left(w_{2} \oplus w_{1}, w^{*}\right)\right)$.

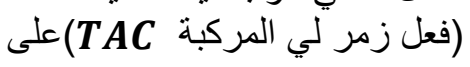

و هذا TAC هوتمثيلات ملساء في G ، أن النظريات المقدمة في هذا البحث انثات وبر هنت وجهزت ببعض النتائج كملاحظات و رسوم. 